\title{
Treinamento proprioceptivo e influência no equilíbrio estático e dinâmico na amputação transfemoral: descrição de caso clínico
}

\author{
Proprioceptive training and influence in static and dynamic equilibrium in transfemoral \\ amputation: a case report
}

\author{
Entrenamiento propioceptivo e influencia en el equilibrio estático y dinámico en la \\ amputación transfemoral: descripción de caso clínico
}

\begin{abstract}
Jéssica Silva Dias ${ }^{1}$, André Pacífico de Souza ${ }^{1}$, Aline Izabela Costa Moreira ${ }^{1}$, Danillo Barbosa ${ }^{2}$, Mônica Beatriz Ferreira ${ }^{1}$, Bruno Bonfim Foresti ${ }^{1}$
\end{abstract}

\section{RESUMO}

Introdução: A amputação é um procedimento cirúrgico que consiste na remoção parcial ou total de uma extremidade do corpo. Sua etiologia está associada a inúmeras patologias de origem vasculares, traumáticas, infecciosas e congênitas que contribuem para a perda do equilíbrio. O objetivo deste estudo é descrever relato de caso analisando os efeitos do treinamento proprioceptivo no equilíbrio estático e dinâmico de um indivíduo com amputação transfemoral utilizando como instrumentos de avaliação a escala do equilíbrio de Berg (EBB) e o teste timed get up and go (TUG).Métodos: Foram realizados 11 atendimentos em um circuito proprioceptivo estabelecido pelo terapeuta. Antes da primeira sessão e imediatamente após a última o paciente foi avaliado através da escala de equilíbrio de Berg e, a cada sessão, era realizado ainda uma avaliação pré e pós-intervenção utilizando o TUG teste. Resultados: Observou-se melhora no tempo de execução do TUG teste quando comparado o pré-atendimento da sessão $01 \mathrm{com}$ a sessão 11, houve uma diminuição de $18 \%$ e uma média de 19,08 22,41 segundos. Já comparado o pós-atendimento da sessão 01 com a sessão 11 , houve uma diminuição de $23,8 \%$ com uma média de $16,7 \pm 1,57$ segundos. Na EEB houve melhora em todos os domínios quando comparado o pré e pós-intervenção. Conclusão: $O$ treinamento proprioceptivo foi efetivo na promoção do equilíbrio estático e dinâmico de um paciente com amputação transfemoral.

Palavras-chave: Treinamento Proprioceptivo, Equilíbrio, Amputação.

\begin{abstract}
Introduction: Amputation is a surgical procedure that involves the partial or total removal of one end of the body. Its etiology is associated with innumerable vascular, traumatic, infectious and congenital pathologies that contribute to the loss of balance. The objective of this study is to describe a case report analyzing the effects of proprioceptive training on the static and dynamic balance of an individual with transfemoral amputation using as instruments of evaluation the Berg balance scale (EBB) and the timed get up and go test). Methods: Eleven appointments were made in a proprioceptive circuit established by the therapist. Prior to and immediately after the first session the patient was assessed through the Berg balance scale and at each session a pre and post-intervention evaluation was performed using the TUG test. Results: We observed an improvement in the TUG test run time when compared to pre-treatment of session 01 with session 11, there was a decrease of $18 \%$ and a mean of $19.08 \pm 2.41$ seconds. Comparing post-attendance from session 01 to session 11 , there was a decrease of $23.8 \%$ with an average of $16.7 \pm 1.57$ seconds. In BSE, there was improvement in all domains when compared to pre- and post-intervention. Conclusion: Proprioceptive training was effective in promoting the static and dynamic balance of a patient with transfemoral amputation.
\end{abstract}

Key words: Proprioceptive Training, Balance, Amputation

${ }^{1}$ Centro Universitário do Sul de Minas - Unis - Departamento de Fisioterapia, Cataguases, MG.

2 Universidade Estadual do Centro Oeste do Paraná - Unicentro - Departamento de Fisioterapia - Defisio, Guarapuava - PR. E-mail: danillo.barbosa@hotmail.com

SUBMETIDO EM: 9/2018

ACEITO EM: 10/2018

PUBLICADO EM: $12 / 2018$

REAS/EJCH | Vol. 11 (1) | e110 | DOI: https://doi.org/10.25248/reas.e110.2019 Página 1 de 8 


\section{RESUMEN}

Introducción: La amputación es un procedimiento quirúrgico que consiste en la remoción parcial o total de un extremo del cuerpo. Su etiología está asociada a innumerables patologías de origen vasculares, traumáticas, infecciosas y congénitas que contribuyen a la pérdida del equilibrio. El objetivo de este estudio es describir relato de caso analizando los efectos del entrenamiento propioceptivo en el equilibrio estático y dinámico de un individuo con amputación transfemoral utilizando como instrumentos de evaluación a escala del equilibrio de Berg (EBB) y la prueba timed get up and go (TUG). Métodos: Se realizaron 11 atendimientos en un circuito propioceptivo establecido por el terapeuta. Antes de la primera sesión e inmediatamente después de la última, el paciente fue evaluado a través de la escala de equilibrio de Berg y, en cada sesión, se realizaba una evaluación pre y post-intervención utilizando la TUG prueba. Resultados: Se observó una mejora en el tiempo de ejecución de la TUG prueba cuando comparado el pre-atendimiento de la sesión 01 con la sesión 11, hubo una disminución del 18\% y una media de 19,08 $\pm 2,41$ segundos. En comparación con la post-atención de la sesión 01 con la sesión 11, hubo una disminución del 23,8\% con una media de 16,7 \pm 1,57 segundos. En la EEB hubo mejoría en todos los ámbitos cuando se compara el pre y post-intervención. Conclusión: El entrenamiento propioceptivo fue efectivo en la promoción del equilibrio estático y dinámico de un paciente con amputación transfemoral.

Palabras Clave: Entrenamiento Proprioceptivo, Equilibrio, Amputación.

\section{INTRODUÇÃO}

A amputação é um procedimento cirúrgico que, durante muito tempo, representou a única possibilidade de cirurgia para o homem (MACEDO et al., 2010). Esse termo refere-se à retirada de um órgão, ou parte dele, situado numa extremidade, porém, quando usado isoladamente, ele é entendido como amputação de membros (PEIXOTO et al., 2017). No Brasil à incidência de amputações evidenciada na literatura apresenta controvérsias quanto ao número de amputados, sendo mais significantes em pacientes patológicos vasculares e variando de 2,8 a 43,9 por 100.000 habitantes/ano (CARVALHO, 2003; MINISTÉRIO DA SAÚDE, 2016; FONSECA et al., 2010; CHINI e BOEMER, 2017; ).

As próteses endoesqueléticas são importantes dispositivos que auxiliam as pessoas amputadas no dia a dia promovendo a sua independência funcional e a integração social, elas podem ser utilizadas para todos os níveis de amputação, com exceção as amputações parciais do pé e do tornozelo. Os joelhos mecânicos modulares também exercem influência na biomecânica da marcha e no equilíbrio dos pacientes amputados transfemorais, eles variam desde os mais básicos sendo os monocêntricos com trava até aos policêntricos com unidades hidráulicas e pneumáticas (CARVALHO, 2001; DE LISA et al., 2002; FISCHINGER, 1982).

A propriocepção é o conjunto de informações aferentes provenientes dos receptores das articulações, músculos, tendões e outros tecidos projetados para o sistema nervoso central, influenciando as respostas reflexas e o controle motor voluntário. Contribui para o controle da postura, estabilidade articular e variadas sensações conscientes (CARVALHO, 2003, LEPHART e FU, 2000).

Evidenciado o déficit de equilíbrio, é necessário a verificação do protocolo de tratamento que deve ser direcionado a um melhor controle corporal, visando melhora do equilíbrio, para que dessa forma possibilite ao paciente uma melhor qualidade de vida sem perdas ou prejuízos, devolvendo a autoestima e confiança, se reinserindo ao convívio social e também ao mercado de trabalho (ROSSI, 2013).

Neste contexto o indivíduo amputado de membro inferior pode apresentar dificuldades na manutenção do equilíbrio estático e dinâmico, o que pode gerar quedas, e consequentemente predispondo-o a fratura, sendo a avaliação do equilíbrio um suporte prognóstico para o desenvolvimento de propostas preventivas e terapêuticas, evitando assim, complicações decorrentes do desequilíbrio (BARAÚNA et al., 2006; RAMOS e SALLES; BARAÚNA et al., 2003).

Sendo assim, o objetivo deste estudo é avaliar a influencia do treinamento proprioceptivo sobre o equilíbrio estático e dinâmico de um indivíduo com amputação transfemoral utilizando como instrumentos de avaliação a escala do equilíbrio de Berg (EBB) e o teste timed get up and go (TUG). 


\section{METODOLOGIA E DETALHAMENTO DO CASO}

\section{Tipo de Estudo e Ética}

Trata-se um estudo clínico, controlado, qualiquantitativo, de caso único, desenvolvido no departamento de fisioterapia do Centro Universitário do Sul de Minas - Unis-MG e Centro de Reabilitação Física, sob a coordenação da disciplina de fisioterapia traumato-ortopédica, na clínica escola de fisioterapia, campus II.

O presente estudo foi submetido para apreciação do Comitê de Ética em Pesquisa da Fundação de Ensino e Pesquisa do Sul de Minas - FEPESMIG, obtendo parecer favorável (1.705.359 e CAAE 58091916.0.0000.5111).

\section{Característica do Relato de Caso}

O presente estudo trata-se de um relato de caso de paciente de \pm 35 anos, $\pm 88 \mathrm{~kg}, \pm 1.75 \mathrm{~m}$. O paciente em questão foi vítima de um acidente automobilístico de caminhão, que ocasionou ferimentos graves nos membros inferiores, em especial no membro inferior esquerdo, que por complicações pós acidente foi amputado á nível transfemoral.

Após a cirurgia o paciente ficou hospitalizado por 3 dias, após a alta, o mesmo permaneceu em sua residência por 30 dias, e só procurou serviço de fisioterapia 55 dias após o pós operatório. No setor de fisioterapia o paciente foi avaliado, e as metas a curto, médio e longo prazo foram estabelecidas pela equipe de reabilitação.

A escola da prótese do paciente foi considerada em virtude do tipo de cirurgia, idade do paciente, ocupação, nível de atividade funcional e laboral, bem como a disponibilidade da prótese no serviço público de saúde. O modelo da prótese é endoesquelética transfemoral, com encaixe flexível, joelho mecânico monocêntrico autobloqueante e com pé articulado.

\section{Critérios de Inclusão}

Os critérios de inclusão do paciente nesse estudo foram: estar ciente do comprometimento que o mesmo deveria assumir em relação ao tratamento fisioterapêutico, assinar e entregar o termo de consentimento livre e esclarecido, apresentar boas condições do coto, não apresentar feridas nem fistulas no coto e comparecer em todas as datas agendadas para medida da prótese.

\section{Critérios de Exclusão}

Os critérios de exclusão do paciente nesse estudo foram: não estar ciente do comprometimento que o mesmo deveria assumir em relação ao tratamento fisioterapêutico, não assinar e não entregar o termo de consentimento livre e esclarecido, não apresentar boas condições do coto, apresentar feridas e fistulas no coto e não comparecer em todas as datas agendadas para medida da prótese

\section{Instrumentos de Avaliação}

\section{Escala de equilíbrio de Berg (EBB)}

É uma avaliação funcional de desempenho do equilíbrio, baseada em 14 tarefas comuns do cotidiano, que avaliam o controle postural incluindo equilíbrio estático e dinâmico, tais como alcançar, transferir-se, girar, permanecer-se em pé, levantar-se e também a flexibilidade (BERG $K$ et al., 1989).

A realização das tarefas da EEB é avaliada através da observação das atividades propostas e a sua pontuação que varia de 0 à 4 em cada atividade, totalizando uma pontuação máxima de 56 pontos. Esta avaliação possui a habilidade de predizer o risco de queda do paciente, sendo de 54 a 56 pontos uma probabilidade de 3 a $4 \%$ no risco de quedas, de 46 a 54 pontos um aumento de 6 a $8 \%$ de chances e abaixo de 36 pontos a o risco de quedas é de quase 100\% (WOOLLACOTT, 2003; BERG, 1989; IZQUIERDO, 1999). 


\section{Teste Timed Get Up and Go (TUG)}

Este instrumento-teste tem por finalidade avaliar o equilíbrio dinâmico e as transferências de sentado para a posição em pé, estabilidade na deambulação e mudanças no percurso da marcha sem utilizar estratégias compensatórias (FIGUEIREDO, 2007).

O fisioterapeuta utiliza um cronômetro digital, e registra o tempo em segundos gasto pelo paciente para realizar o teste. É necessária somente uma cadeira com encosto e apoio para os braços e um espaço de três metros com referência livre de obstáculos. O paciente é orientado a se levantar da cadeira, caminhar uma distância de três metros, dar meia volta, retornar para a cadeira e sentar-se novamente, neste caso fazendo uso de uma prótese transfemoral endoesquelética (MORAES, 2008; CUNHA, 2006).

\section{Protocolo de Intervenção}

O protocolo de atendimento foi composto por três sessões de fisioterapia semanais durante um período de 12 semanas totalizando 36 sessões de fisioterapia, tendo a duração de 50 minutos em média. Foi registrada apenas 1 falta durante os tratamentos. A escala de equilíbrio de Berg e o teste timed get up foi aplicada antes da $1^{\text {a }}$ sessão, na $20^{\text {a }}$ sessão e após a última sessão, sempre pelo mesmo avaliador.

\section{O protocolo fisioterapêutico foi dividido em 4 fases:}

I Fase: era solicitado ao paciente fazer o circuito proposto, passando por cada obstáculo que contava com: circuito proprioceptivo, trampolim, balanço proprioceptivo, placa proprioceptiva e barra paralela

II. Fase: o paciente era orientando á andar na barra paralela, por uma distância de 2.40 metros, fazer a transferência para escada de canto, e percorrer uma distância de 5.40 metros, mantendo a concentração, equilíbrio, sinergismo músculo-esquelético e coordenação motora.

III. Fase: o paciente era orientado á caminhar por 4 metros numa velocidade de $2.0 \mathrm{~km}$ sem apoio, além de realizar os deslocamentos laterais, mantendo a postura e alinhamento biomecânico.

IV. Fase: o paciente foi orientado a realizar 8 voltas completas no circuito, mantendo o mesmo padrão de qualidade exigido nas demais fases do protocolo. Após o término o paciente recebia uma atenção especial quanto aos sinais vitais (pressão arterial e ritmo respiratório), além de ser reavaliado.

O paciente recebeu orientações iniciais sobre como realizar cada exercício, respeitando suas limitações físicas, mas foi solicitado ao paciente que realizasse o circuito com a máxima perfeição biomecânica, para que não houvesse sobrecarga nas outras articulações. O paciente realizou 3 repetições em cada equipamento, com diminuição de 1 minuto entre cada passagem.

\section{Descrição do Protocolo - Circuito}

Abaixo (Figura 1) paciente realizando a ambientação do circuito, para isso ele foi orientado á fazer uma simulação onde o mesmo subiria e desceria somente $01^{\circ}$ degrau da escada várias vezes, até ganhar confiança para fazer todo circuito. Observa-se na seta 1, o alinhamento do membro inferior protetizado, na posição inicial em extensão completa, pronto para fazer a semi-flexão e realizar o movimento desejado, já na seta 2 e 3 apontam as distancia de cada degrau $(30 \mathrm{~cm})$, bem como a altura do corrimão $(1.10 \mathrm{~m})$, essas informações tornam-se importantes para replicação do trabalho, bem como garantir que a pesquisa seja controlada em todas as fases, garantindo ao paciente segurança e o maior aproveitamento motor, sem causar desconforto e coloca-lo em situação de risco de quedas.

A figura 2 apresenta o paciente realizando uma parte do circuito planejado e controlado, a seta 1 aponta o alinhamento corporal sugerido para o deslocamento, seta 2 aponta a altura da barra paralela $(75 \mathrm{~cm}$ de altura), a seta 3 aponta a distância entra as barras paralelas $(85 \mathrm{~cm})$, a seta 4 aponta a divisão central onde 0 paciente que tem manter-se posicionado, a fim de manter o equilíbrio corporal e a seta 5 aponta a distância média percorrida pelo paciente $(5.5 \mathrm{~m})$ numa velocidade média de $2.0 \mathrm{~km}$. 


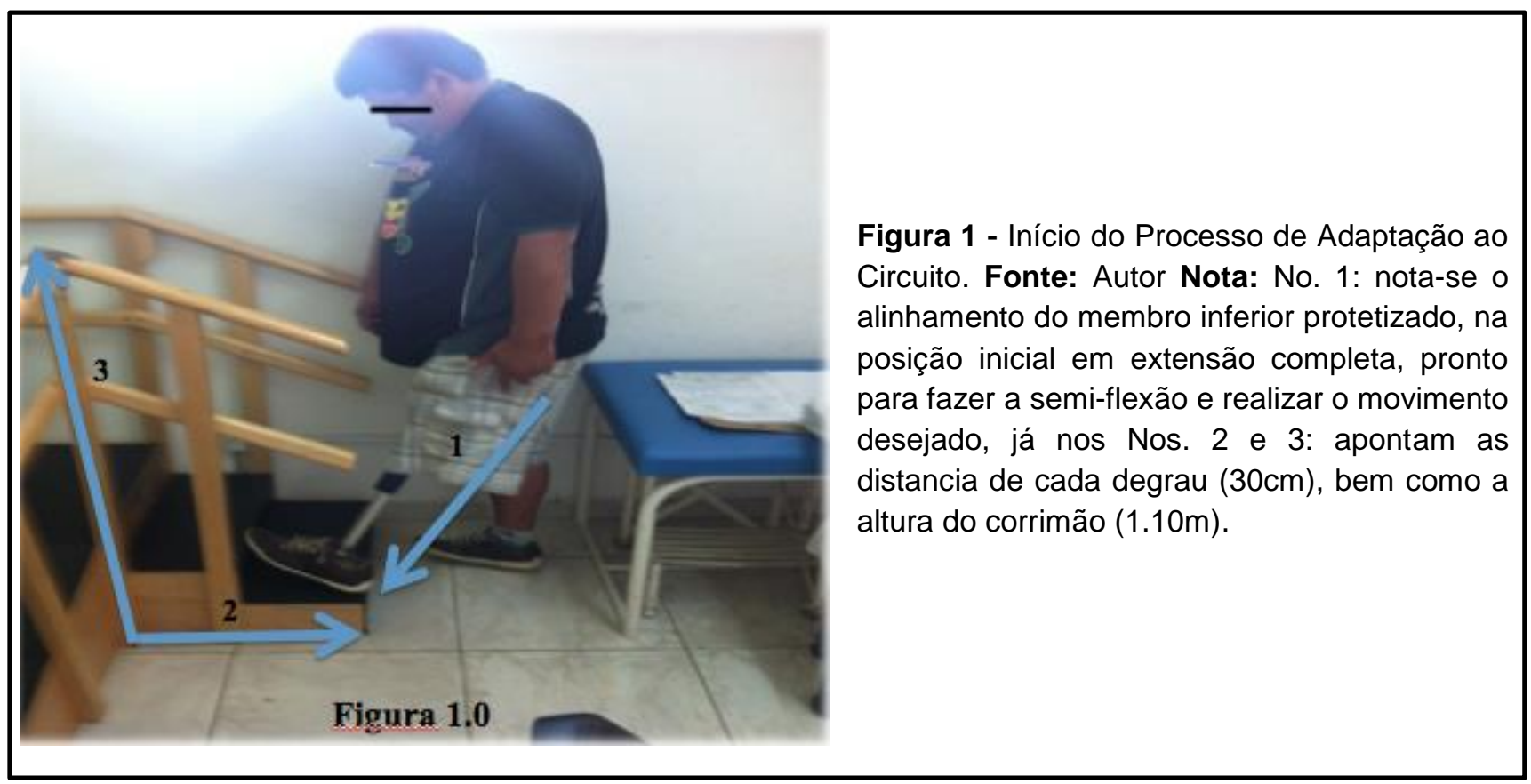

Os dados foram inseridos na planilha do Microsoft Excel, onde foram calculados as médias, desvio padrão e o valor de $P<0,05$ de todas as variáveis, em seguido pela análise de comparação múltipla entre os valores dos testes (Tukey's), sendo considerado significativo os valores de $p<0,5$ que foi realizado no Grap Prisma.

\section{Procedimentos para Análise dos Dados}

Os dados foram inseridos na planilha do Microsoft Excel, onde foram calculados as médias, desvio padrão e o valor de $P<0,05$ de todas as variáveis, em seguido pela análise de comparação múltipla entre os valores dos testes (Tukey's), sendo considerado significativo os valores de $p<0,5$ que foi realizado no Grap Prisma.

Figura 2 - Paciente iniciando o circuito proprioceptivo, com o auxílio de barras paralelas. Fonte: Autor. Nota: No. 1: aponta $\circ$ alinhamento corporal sugerido para o deslocamento, No. 2: aponta a altura da barra paralela $(75 \mathrm{~cm}$ de altura), No. 3 : aponta a distancia entra as barras paralelas $(85 \mathrm{~cm})$, No. 4: aponta a divisão central onde o paciente que tem manter-se posicionado, a fim de manter o equilíbrio corporal e o No. 5: aponta a distância média percorrida pelo paciente $(5.5 \mathrm{~m})$.

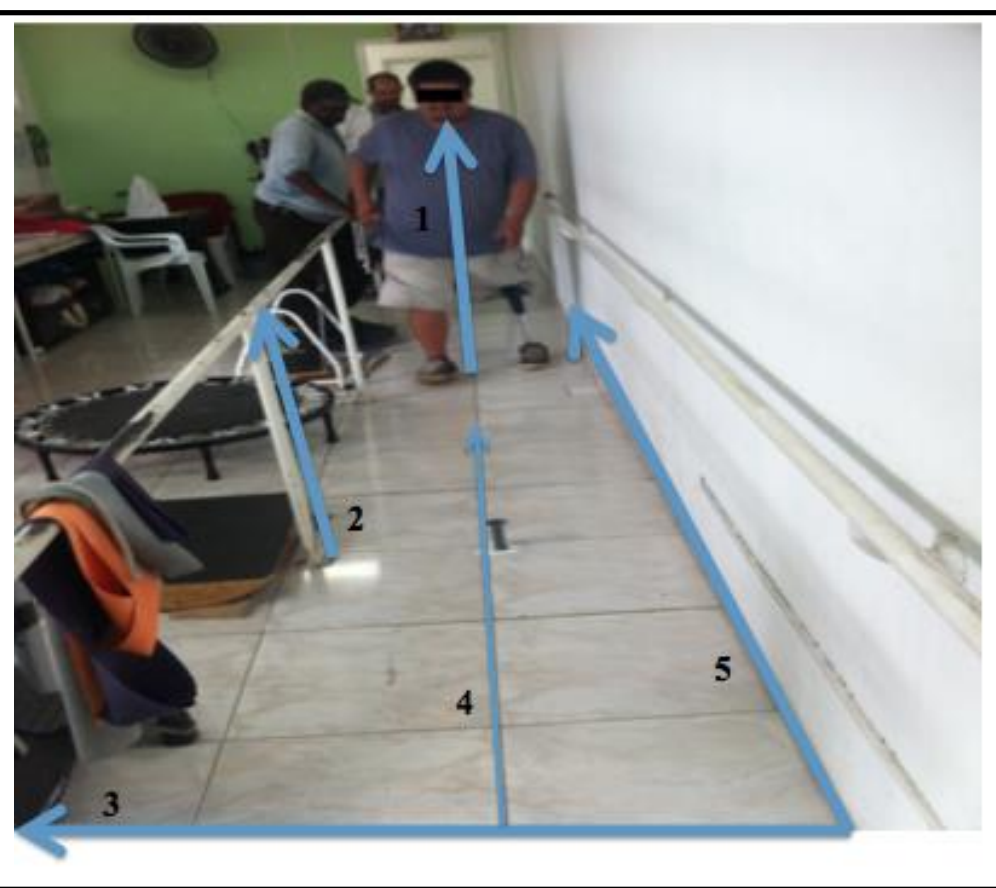




\section{RESULTADOS}

No presente relato de caso de caso, foi avaliado o desempenho do paciente através do teste Timed Up Go (TUG), para analisar o equilíbrio do paciente em três momentos diferentes, antes da aplicação do protocolo de fisioterapia (Timed Up Go - Pré Fisiot), na $20^{\circ}$ sessão de fisioterapia (Timed Up Go - 20 Fisiot) e após a $35^{\circ}$ sessão de fisioterapia (Timed Up Go Pós Fisiot). O teste foi realizado 3 vezes e obtido um valor médio. Os achados evidenciaram uma queda substancial no score nos 3 momentos da avaliação, a média do score do time up go pré fisioterapia foi de 34 segundos, no timed up go na $20^{\circ}$ sessão foi de 24.6 segundos e no timed up go pós fisioterapia foi de 16.3 segundo. Sugere-se para estes teste que o tempo seja inferior a 20 segundos, o que corresponde por sua vez a um baixo risco de quedas, quando o valor se encontra entre 20 a 29 segundo, considera-se um risco médio ou intermediário de queda, e para um tempo de 30 segundos ou mas, considera-se um alto risco para quedas (Gráfico 1).

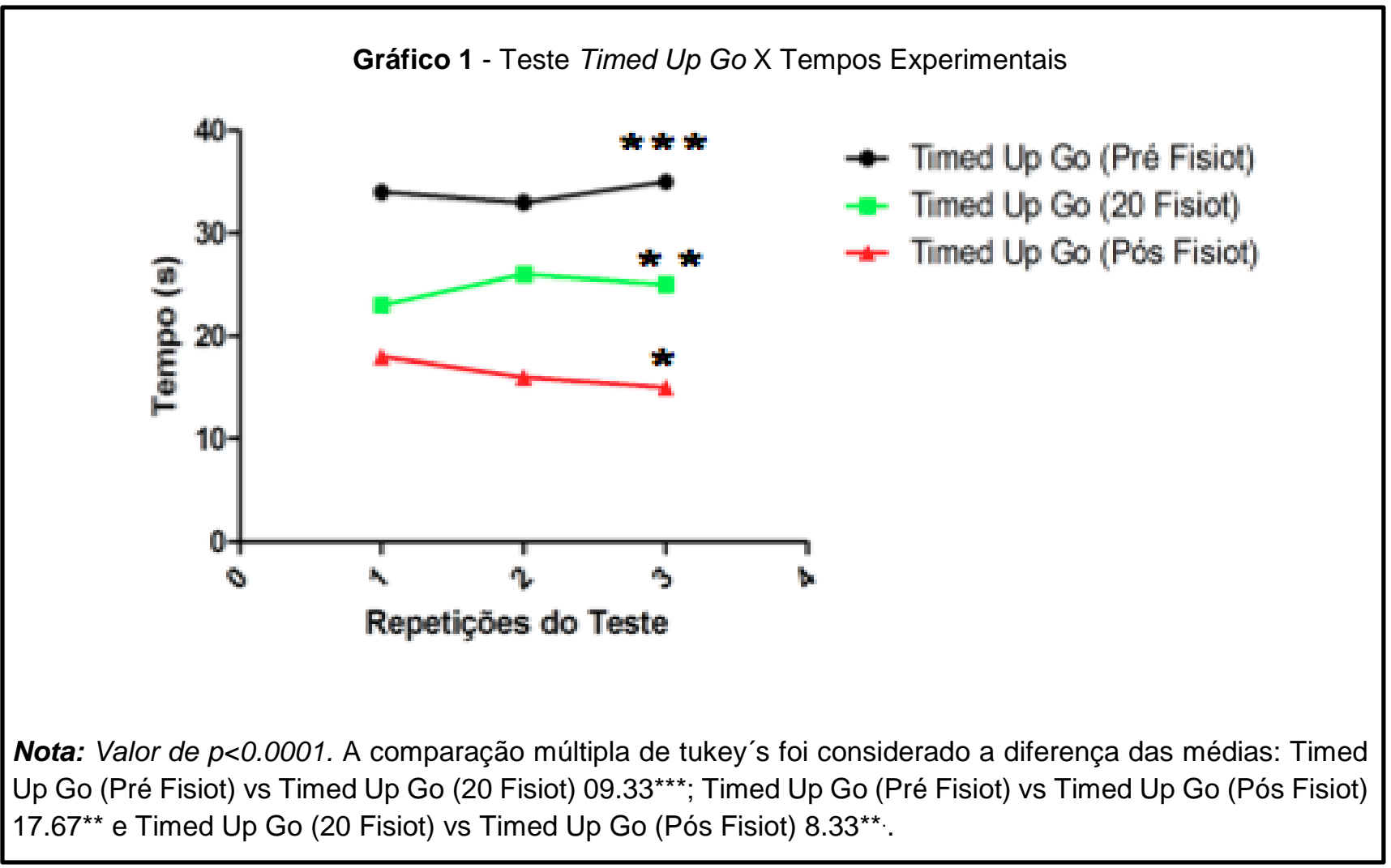

Observou-se no teste de equilíbrio de Berg realizado em 3 momentos: berg pré tratamento de fisioterapia, berg na $20^{\circ}$ sessão de fisioterapia e berg pós tratamento de fisioterapia. O teste foi realizado 3 vezes e obtido um valor médio. Nota-se que o berg pré tratamento resultou no valor médio de 47.3 pontos, já o berg na $20^{\circ}$ sessão de fisioterapia apontou 32 pontos e no berg pós tratamento de fisioterapia observou o valor de 21 pontos (Gráfico 2).

A escala de equilíbrio de berg representa uma forma segura e validada para analisar o equilíbrio estático do paciente, bem como analisar de forma objetiva o desenvolvimento do paciente em função das intervenções propostas, no sentido, nota-se que o protocolo proposto para o paciente amputado resultou em melhora do nível de equilíbrio, promovendo mas segurança e conforto na deambulação. A escala de equilibrio e berg preconiza um score máximo de 56 pontos, o que representa $100 \%$ de chance de queda. 


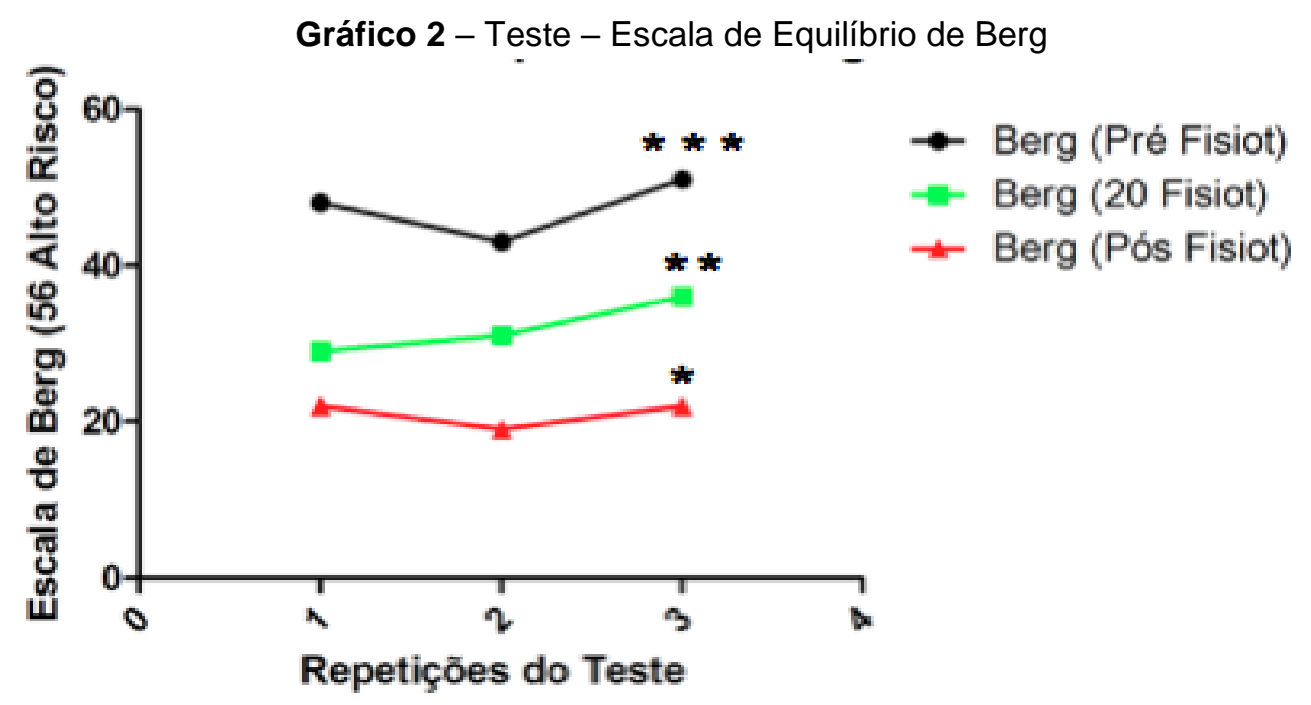

Nota: Valor de $p<0.0002$. A comparação múltipla de tukey's foi considerado a diferença das medias: Berg (Pré Fisiot) vs Berg (20 Fisiot) 15,33**, Berg (Pré Fisiot) vs Berg (Pós Fisiot) $26,33^{\star *}$ e Berg (20 Fisiot) vs Berg (Pós Fisiot) 11.0*.

\section{DISCUSSÃO}

A reabilitação e a promoção do equilíbrio em indivíduos amputados unilaterais é baseada na exposição frequente do amputado às variações de instabilidade que ocorrem repetidamente e paralela às condições de dificuldades posturais com intuito da promoção de novas habilidades (ABREU, 2008; ZAMBALDI, 2007)

Os resultados obtidos neste estudo demonstram que a diminuição das oscilações posturais que representa o maior risco de queda ao indivíduo amputado ocorreu nas tarefas da EEB principalmente na fase pósintervenção e corrobora também com os achados do teste timed get up and go pós-atendimento comprovando que o indivíduo amputado quando exposto a condições de dificuldades melhora o seu equilíbrio estático. Há evidências de que exercícios proprioceptivos direcionados para a estimulação do equilíbrio através do sistema visual, sistema vestibular e sistema somatossensorial mostram-se eficazes na manutenção do equilíbrio estático e dinâmico e no processo de adaptação à prótese (JAGS, 2011; SOUZA, 2009).

Após o protocolo de intervenção proprioceptiva, o participante deste estudo apresentou um aumento no score total da avaliação pré-intervenção para pós-intervenção da escala de berg de 47.3 para 21 pontos, resultando em uma melhora na condição do equilíbrio estático e dinâmico do paciente, levando a uma diminuição na possibilidade de queda.

Santos et al. (2009) relatam que quanto menor o desempenho na EEB, maior é a probabilidade de queda em amputados transfemorais unilaterais, e confirmam que um amputado transfemoral com uma pontuação inferior a 36 pontos tem aproximadamente dez vezes mais chances de sofrer queda do que aqueles com pontuação igual ou superior (SOARES, 2008).

A redução da velocidade de deambulação no teste timed get up and go no amputado usuário de prótese transfemoral, tem sido associada a uma diminuição da capacidade do indivíduo em controlar o equilíbrio corporal durante a realização de uma tarefa de dificuldade mínima (ABREU, 2008).

No presente estudo observou-se que houve uma melhora significativa no risco de quedas em ambas as fases da avalição pré e pós-intervenção com diminuição do tempo de realização do TUG teste e de 34 para 20 segundos. 
Kofotolis et al. (2003) avaliaram os efeitos do treinamento proprioceptivo no equilíbrio de um paciente amputado transfemoral unilateral. Eles randomizaram a aplicação de dois testes: o teste timed get up and go (TUG) e a escala do equilíbrio de Berg (EEB), e, ao final de quatro semanas, observaram uma melhora significativa tanto na velocidade da marcha quanto na melhora do treinamento proprioceptivo como na adaptação da prótese transfemoral.

Sherrington et al. (1999), em sua revisão sistemática sobre exercícios proprioceptivos e prevenção de quedas em amputados transfemorais, formularam recomendações cujos exercícios tinham como objetivo desafiar o equilíbrio estático e dinâmico através da redução progressiva da base de apoio bipodal, transferir o peso do corpo de uma perna para outra e, a adaptação à nova prótese.

\section{CONSIDERAÇÕES FINAIS}

O treinamento proprioceptivo foi efetivo na promoção do controle postural no indivíduo amputado unilateral transfemoral usuário de prótese endoesquelética, interferindo diretamente na evolução do equilíbrio estático e dinâmico, minimizando o risco de cair, prevenindo quedas e favorecendo a independência motora e funcional nas atividades diárias.

\section{REFERÊNCIAS}

1. ABREU F. Estudo comparativo entre a avaliação do equilíbrio estático de indivíduos amputados e não amputados [Tese Doutorado em Motricidade Humana]. Lisboa(Portugal): Universidade Técnica de Lisboa; 2008, p.22-9.

2. BARAÚNA MA, BARBOSA SRM, CANTO RST et al. Estudo do equilíbrio estático de idosos e sua correlação com quedas. Revista Brasileira de Fisioterapia, 2006, 17(2): 136-41.

3. BARAÚNA MA. Avaliação do equilíbrio estático do portador de diabetes mellitus pela biofotogrametria. Diabetes Clínica, 2003, 7(1): 3-8.

4. BERG K, WOOD-DAUPHINÉE S, WILLIAMS JI. Measuring balance in the elderly: preliminary development of an instrument. Journal Physiotherapy, 1989, 2(1): :304-11.

5. CARVALHO JA. Amputações de membros inferiores: em busca de plena reabilitação. 2ª ed., São Paulo, SP, Manole, 2003, p. 12242.

6. CARVALHO SD. Amputações de membros inferiores. 1ํㅡ. Eão Paulo, SP, Manole 2001, p.34-9.

7. CHINI GCO, BOEMER MR. Amputação na percepção de quem a vivencia: um estudo sob a ótica fenomenológica. Rev Latino-Am Enfermagem, 2007; 15(2): 330-6.

8. CUNHA J. The timed get up and go test revisited: measurement of the component tasks. J Rehabil Research Dev, 2006, 37(1): 10914.

9. DE LISA J. Tratado de Medicina de Reabilitação. 3. ed. Barueri, SP, Editora Manole, 2002, p.77-90.

10. FIGUEIREDO JS. Brazilian version of the Berg balance scale. Braz. Med. Biol. Res, 2007, 37(9): 1411-21.

11. MARTINS RO. A prática do iso-stretching na melhora da percepção corporal [monografia]. Cascavel: Unioeste; 2004., p. 22-33.

12. FONSECA PHS, RODRIGUES CO, VANZ FO. Método Iso-stretching na otimização das aptidões para a prática do futebol de campo. Rev Saúde. 2010; 12(2); 57-64.

13. IZQUIERDO RW. Long-term reliability of the timed up-and-go test among community-dwelling elders. Journal of Physical Therapy Science, 17(1): 93-96.

14. JAGS I. The functional assessment Berg Balance Scale is better capable of estimating fall risk in the elderly than the posturographic Balance Stability System. Arq Neuropsiquiatr., 2011, 71(1) :5-11.

15. BALOH RW, FIFE TD, ZWELING L et al. Comparison of static and dynamic posturography in young and older normal people. J Am Geriatr Soc, 1994, 42(4): 4, p.405-12.

16. LEPHART SM, FU FH. Proprioception and neuromuscular control in joint stability. Champaigne (IL): HumanKinetics, 2000, p. 2-18.

17. MACEDO CSG, DEBIAGI PC, ANDRADE FM. Efeito do isostretching na resistência muscular de abdominais, glúteo máximo e extensores de tronco, incapacidade e dor em pacientes com lombalgia. Fsioterapia em Movimento, 2010; 23(1): 113-20.

18. Ministério da Saúde. Datasus. Informações de Saúde (Tabnet). Brasília, DF; 2016. [cited 2016 Apr 18]. Available from: Available from: https://goo.gl/pRDToU

19. MORAES EN. Avaliação multidimensional do idoso - instrumentos de rastreio. Belo Horizonte, MG Folium, 2008, p. 25-55.

20. PEIXOTO AM, ZIMPEL SA, OLIVEIRA ACA et al. Prevalência de amputações de membros superiores e inferiores no estado de Alagoas atendidos pelo SUS entre 2008 e 2015. Fisioterapia e Pesquisa, 2017; 24(4): 378-84.

21. RAMOS S. Evaluation of the static balance lower limb amputeers by means of computerized biophotogrammetry. Revista Brasileira de Fisioterapia, 2005, 10(1): 1-9.

22. ROSSI AG. Reabilitação Vestibular e Posturografia Dinâmica. $1^{\text {a }}$ ed. Santa Maria, RS 2003, p. 7-19.

23. SANTOS BB. Apliçção da Escala de Equilíbrio de Berg para verificação de equilíbrio de idosos em diferentes fases do envelhecimento. Rev. Bras. Ciência do Envelhecimento Humano, 2009, (8): 4-9.

24. SHERRINGTON RF, PAUL HJ, FANTER KJ et al. Quedas em idosos: fatores de risco associados. Gerontologia, $1999,7(1): 15-21$.

25. SOARES S. The Timed Get Up \& Go: A test of basic functional mobility for frail elderly persons. J A. Geriatr Soc, 2008, 39(1):142-8.

26. SOUZA H. Caracterização clínico-funcional do equilíbrio em idosos portadores de Diabetes Mellitus do tipo II. [Dissertação]. São Paulo: Universidade Federal de São Paulo / Escola Paulista de Medicina, 2009.

27. WOOLLACOTT H. Controle motor: teoria e aplicações práticas. São Paulo, SP: Manole, 2003, p. 21-39.

28. ZAMBALDI A. Study of elderly static balance and its correlation with falls. Revista Brasileira de Fisioterapia, 2007, 8(2): 10-7. 